\title{
VECTOR-VALUED CONTINUOUS FUNCTIONS WITH STRICT TOPOLOGIES AND ANGELIC TOPOLOGICAL SPACES
}

\author{
SURJIT SINGH KHURANA
}

\begin{abstract}
It is proved that if $X$ is a metric space, $E$ a Banach space containing a $\sigma$-weakly-compact dense subset, then the space $\left(M_{\tau}\left(X, E^{\prime}\right)\right.$, $\left.\sigma\left(M_{\tau}\left(X, E^{\prime}\right), C_{b}(X, E)\right)\right)$ is angelic, $C_{b}(X, E)$ being all bounded continuous functions from $X$ into $E$ and $M_{\tau}\left(X, E^{\prime}\right)$ the dual of $C_{b}(X, E)$ with the strict topology $\beta$.
\end{abstract}

A Hausdorff topological space $Y$ is called angelic if (i) every relatively countably compact subset of $Y$ is relatively compact, and (ii) for any point $x$ in the closure of a relatively compact subset $A$ of $Y$, there exists a sequence, in $A$, converging to $x[4, \mathrm{p}$. 534]. In this paper $X$ will stand for a completely regular Hausdorff space, $E$ a Banach space over $K$, the field of real or complex numbers, $C(X, E)(C(X))$ all $E$-valued ( $K$-valued) continuous functions on $X$, and $C_{b}(X, E)\left(C_{b}(X)\right)$ all bounded $E$-valued ( $K$-valued) continuous functions on $X$. We shall use the notations of [5] for locally convex spaces. Also the notations and results from [2] will be used. The topologies $\beta, \beta_{0}, \beta_{1}, \beta_{e}$ (also denoted by $\beta_{\infty}$ ) are defined on $C_{b}(X)$ in [9], [7], [8]-these topologies are defined for $K=R$, the reals, but naturally extend to $K=\mathrm{C}$, the complex field. $\beta, \beta_{0}, \beta_{1}, \beta_{\infty}$ are defined on $C_{b}(X, E)$ in [1], [2]. If $\mathcal{H}^{\infty}=\mathcal{H}^{\infty}(X, E)=\left\{H \subset C_{b}(X, E): H\right.$ pointwise equicontinuous and uniformly bounded $\}$, the topology $\beta_{\infty}$ is the finest locally convex topology on $C_{b}(X, E)$ agreeing with pointwise topology on each $H \in \mathcal{H}^{\infty}$. It is well known that $M_{\tau}\left(X, E^{\prime}\right)=\left(C_{b}(X, E), \beta\right)^{\prime}, M_{t}\left(X, E^{\prime}\right)=\left(C_{b}(X, E), \beta_{0}\right)^{\prime}$ and $M_{\infty}\left(X, E^{\prime}\right)=\left(C_{b}(X, E), \beta_{\infty}\right)^{\prime}$ (see [2]). If $X$ is a metric, $M_{\infty}\left(X, E^{\prime}\right)=$ $M_{\tau}\left(X, E^{\prime}\right)$ and $\beta$ and $\beta_{\infty}$ are both Mackey [2]; from this it follows that $\beta=\beta_{\infty}$ in this case.

If $X$ is a metric space it is proved in [5, Theorem 5.3] that $\left(M_{\infty}(X), \sigma\left(M_{\infty}(X), C_{b}(X)\right)\right)$ is angelic. We will extend this result to $M_{\infty}\left(X, E^{\prime}\right)$ for some special Banach spaces $E$.

THEOREM 1. $\left(F^{\prime}, \tau\left(F^{\prime}, F\right)\right)$ is complete, $\tau$ denoting the Mackey topology of the duality $\left\langle F, F^{\prime}\right\rangle$, where $F=C_{b}(X, E)$ and $F^{\prime}=M_{\infty}\left(X, E^{\prime}\right)$.

Proof. By Grothendieck completeness theorem [5, Theorem 6.2, p. 148], it is enough to prove that any linear form $\mu$ on $F$ which is continuous on every

Received by the editors May 6, 1977 and, in revised form, August 8, 1977.

AMS (MOS) subject classifications (1970). Primary 46G10; Secondary 28A45, 54E99.

(1) American Mathematical Society 1978 
absolutely convex compact subset $\left(F, \sigma\left(F, F^{\prime}\right)\right)$ with topology induced by $\beta_{\infty}$, belongs to $F^{\prime}$. Considering $\mu:\left(C_{b}(X, E),\|\cdot\|\right) \rightarrow K$, we first prove that $\|\mu\|<\infty$. Since a norm convergent sequence has a compact absolutely closed convex hull, we get $\|\mu\|<\infty$ (standard argument). Fix an $x \in E$ and an $H \in \mathcal{H}^{\infty}(X, K), H$ absolutely convex and closed with pointwise topology. $H$ is $\sigma\left(C_{b}(X), M_{\infty}(X)\right)$-compact [2], [3]. From this it easily follows $H \otimes x$ is $\sigma\left(F, F^{\prime}\right)$-compact and absolutely convex and so $\mu_{x} \in M_{\infty}(X)$ which implies that $\mu \in M_{\infty}\left(X, E^{\prime}\right)[2]$.

THEOREM 2. If $X$ is a complete metric space, then $\beta=\beta_{\infty}=\beta_{0}$.

Proof. Since $X$ is a complete metric space, $M_{t}\left(X, E^{\prime}\right)=M_{\tau}\left(X, E^{\prime}\right)=$ $M_{\infty}\left(X, E^{\prime}\right)$. Also $\beta$ and $\beta_{\infty}$ are both Mackey [2]. From this we get $\beta=\beta_{\infty}$. Let $P$ be an absolutely convex compact subset of $\left(F^{\prime}, \sigma\left(F^{\prime}, F\right)\right)$, where $F=C_{b}(X, E)$ and $F^{\prime}=M_{\infty}\left(X, E^{\prime}\right)$. This means $|P|$ is relatively compact in $\left(M_{\tau}(X), \sigma\left(M_{\tau}(X), C_{b}(X)\right)\right)$ [2, Proof of Theorem 3.7]. When $E=K$ and $X$ is a complete metric space, $\beta=\beta_{0}\left[7\right.$, Theorem 5.8(a)] and so $|P|$ is $\beta_{0}$-equicontinuous. Thus, given $\varepsilon>0$, there exists a compact $K \subset X$ such that $|\mu|(X \backslash K)<\varepsilon, \forall \mu \in P$. This proves $P$ is $\beta_{0}$-equicontinuous [3, Lemma 2].

THEOREM 3. Let $X$ be a metric space and suppose $E$ contains a $\sigma$-weaklycompact dense subset. Then $\left(F^{\prime}, \sigma\left(F^{\prime}, F\right)\right)$ is an angelic space, where $F=$ $C_{b}(X, E), F^{\prime}=M_{\infty}\left(X, E^{\prime}\right)$.

Proof. Since $\left(F^{\prime}, \tau\left(F^{\prime}, F\right)\right)$ is complete, relative countable compactness implies relative compactness in $\left(F^{\prime}, \sigma\left(F^{\prime}, F\right)\right)$ [5, Theorem 11.2, p. 187]. Let $\tilde{X}$ be the completion of $X$ and let $K_{0}$ be a relative compact subset of $\left(F^{\prime}, \sigma\left(F^{\prime}, F\right)\right)$ and $\lambda_{0} \in K_{1}=$ closure of $K_{0}$. Every $\mu \in M_{\tau}\left(X, E^{\prime}\right)$ gives rise to $\tilde{\mu} \in M_{\tau}\left(\tilde{X}, E^{\prime}\right), \tilde{\mu}(g)=\mu\left(\left.g\right|_{X}\right), \forall g \in C_{b}(\tilde{X}, E)$. Thus $\tilde{K}_{1}$ is compact in $\left(F_{1}^{\prime}, \sigma\left(F_{1}^{\prime}, F_{1}\right)\right)$, where $F_{1}=C_{b}(\tilde{X}, E)$ and $F_{1}^{\prime}=M_{\tau}\left(\tilde{X}, E^{\prime}\right)$. Since $\beta_{\infty}=\beta=$ $\beta_{0}$ on $C_{b}(\tilde{X}, E)$ and $\beta_{\infty}$ is strongly Mackey, there exists an increasing sequence $\left\{D_{n}\right\}$ of compact subsets of $\tilde{X}$ such that $|\mu|\left(\tilde{X} \backslash \cup_{n=1}^{\infty} D_{n}\right)=0$, $\forall \mu \in \tilde{K}_{1}$. Also since $F_{2}=C_{b}(\tilde{X}) \otimes E$ is dense in $\left(F_{1}, \sigma\left(F_{1}, F_{1}^{\prime}\right)\right)$, the topologies $\sigma\left(F_{1}^{\prime}, F_{1}\right)$ and $\sigma\left(F_{1}^{\prime}, F_{2}\right)$, restricted to $\tilde{K}_{1}$, coincide [2].

Let $X_{1}=\operatorname{cl}\left(\cup D_{n}\right)$ in $\tilde{X}$, and let $X_{2}$ be a compact metric space in which $X_{1}$ is densely embedded (note $X_{1}$ is a separable metric space). For a $\tilde{\mu} \in \tilde{K}_{1}$, $f \in C\left(X_{2}\right)$ and $x \in E$, define $\tilde{\tilde{\mu}} \in M_{\tau}\left(X_{2}, E^{\prime}\right), \tilde{\tilde{\mu}}(f \otimes x)=\tilde{\mu}\left(f_{1} \otimes x\right)$, where $f_{1}$ is any continuous extension of $\left.f\right|_{X_{1}}$ to $\tilde{X}$ with $\left\|f_{1}\right\|=\|f\|$ (sup norm) (this is possible by the Tietze extension theorem; also $\tilde{\tilde{\mu}}$ is well defined). Since $C\left(X_{2}\right)$ is separable in norm topology and $C\left(X_{2}\right) \otimes E$ separates the points of $\tilde{\tilde{K}}_{1}=$ $\left\{\tilde{\tilde{\mu}}: \mu \in K_{1}\right\}$, there exists a countable subset $P_{1}$ of the unit ball of $C\left(X_{2}\right)$ such that $P \otimes E$ separates points of $\tilde{\tilde{K}}_{1}$ (note $P \otimes E=\{p \otimes x: p \in P, x \in E\}$ [5]). This gives a countable set $P_{2}$ in the unit ball of $C_{b}(\tilde{X})$ such that $P_{2} \otimes E$ separates points of $\tilde{K}_{1}$. So we get a countable set $P$ in the unit ball of $C_{b}(X)$ such that $P \otimes S$ separates the points of $K_{0}, S$ being the closed unit ball of $E$. Giving $P$ the discrete topology and $S$ the topology induced by the weak topology on $E$, we see that $P \times S$, with product topology, has a $\sigma$-compact 
dense subset. Also for any $f \in C_{b}(X)$ and $\mu \in M_{\tau}\left(X, E^{\prime}\right), \mu(f \otimes \cdot) \in E^{\prime}$ and so every $\mu \in M_{\infty}\left(X, E^{\prime}\right)$ is continuous on $P \times S$. Let $\left\{\mu_{\alpha}\right\}$ be a net in $K_{0}$ such that $\mu_{\alpha} \rightarrow \lambda_{0}$, pointwise on $C_{b}(X, E)$. By [4, Theorem 0.1$]$ there exists a sequence $\left\{\mu_{n}\right\} \subset\left\{\mu_{\alpha}\right\}$ such that $\mu_{n} \rightarrow \lambda_{0}$ on $P \times S$. This implies $\mu_{n} \rightarrow \lambda_{0}$ on $C_{b}(X, E)$, since $K$ is compact and $P \times S$ separates the points of $K$. This proves the result.

I am grateful to the referee for useful suggestions.

\section{REFERENCES}

1. R. A. Fontenot, Strict topologies for vector-valued functions, Canad. J. Math. 26 (1974), 841-853.

2. S. S. Khurana, Topologies on spaces of vector-valued continuous functions, Trans. Amer. Math. Soc. (to appear).

3. S. S. Khurana and S. A. Choo, Strict topology and P-spaces, Proc. Amer. Math. Soc. 61 (1976), 280-284.

4. J. D. Pryce, $A$ device of $R$. J. Whitley applied to pointwise compactness in spaces of continuous functions, Proc. London Math. Soc. 23 (1971), 532-546.

5. H. H. Schaefer, Topological vector spaces, Macmillan, New York, 1966.

6. J. Schmets and J. Zafarani, Topologie stricte faible et mesures discrètes, Bull. Soc. Roy. Liège 43 (1974), 405-418.

7. F. D. Sentilles, Bounded continuous functions on completely regular spaces, Trans. Amer. Math. Soc. 168 (1972), 311-336.

8. F. D. Sentilles and R. F. Wheeler, Linear functionals and partition of unity in $C_{b}(X)$, Duke Math. J. 41 (1974), 483-496.

9. R. F. Wheeler, The strict topology, separable measures and paracompactness, Pacific J. Math. 47 (1973), 287-302.

Department of Mathematics, University of Iowa, Iowa City, Iowa 52242 